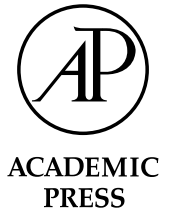

\title{
Brazil and the development of international scientific biosafety testing guidelines for transgenic crops
}

\author{
Deise Maria Fontana Capalbo, ${ }^{\mathrm{a}, *}$ Angelika Hilbeck, ${ }^{\mathrm{b}}$ David Andow, ${ }^{\mathrm{c}}$ Allison Snow, ${ }^{\mathrm{d}}$ \\ Bui Ba Bong, ${ }^{\mathrm{e}}$ Fang-Hao Wan, ${ }^{\mathrm{f}}$ Eliana M.G. Fontes, ${ }^{\mathrm{g}}$ Ellie Onyango Osir, ${ }^{\mathrm{h}}$ Gary P. Fitt, ${ }^{\mathrm{i}}$ \\ Jill Johnston, ${ }^{\mathrm{d}}$ Josephine Songa ${ }^{\mathrm{j}}$ K.L. Heong, ${ }^{\mathrm{k}}$ and A. Nicholas E. Birch ${ }^{1}$

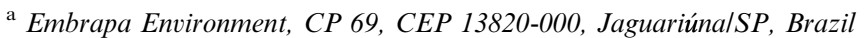 \\ ${ }^{\mathrm{b}}$ Swiss Federal Institute of Technology, Geobotanical Institute, Zurich, Switzerland \\ ${ }^{\mathrm{c}}$ Department of Entomology, University of Minnesota, St. Paul, USA \\ d Ohio State University, Columbus, USA \\ e Vietnamese Ministry of Agriculture and Rural Development, Hanoi, Viet Nam \\ ${ }^{\mathrm{f}}$ Chinese Academy of Agricultural Sciences, Beijing, China \\ g Embrapa Genetic Resources and Biotechnology, Brasilia, Brazil \\ ${ }^{\mathrm{h}}$ International Centre of Insect Physiology and Ecology, Nairobi, Kenya \\ ${ }^{i}$ Australian Cotton Cooperative Research Center, Narrabri, Australia \\ ${ }_{\mathrm{j}}$ National Dryland Farming Research Centre, Katumani, Kenya \\ ${ }^{\mathrm{k}}$ International Rice Research Institute, Metro Manila, Philippines \\ ${ }^{1}$ Scottish Crop Research Institute, Dundee, Scotland, UK \\ Received 2 January 2003; accepted 28 February 2003
}

\begin{abstract}
Under the umbrella of the International Organisation of Biological Control (IOBC), an international working group of public sector scientists entitled on "Transgenic Organisms in Integrated Pest Management and Biological Control" has been organized. The group will develop scientific principles and detailed scientific guidelines for biosafety testing of transgenic crops. The key elements of this project are: (1) An international initiative including expert scientists from leading research institutions in developed and developing countries; (2) coordination of the development and implementation of the guidelines as a dynamic process, which will include scientific and technical capacity building and communication among scientists and between scientists and policy makers; (3) rapid serial publication of sections of the guidelines as they are completed; and (4) rapid and timely revision of previously published sections. The guidelines will be constructed on a case-by-case basis and will have no regulatory legitimacy themselves. (C) 2003 Elsevier Science (USA). All rights reserved.
\end{abstract}

Keywords: Genetically modified plants; Biosafety; Guidelines; Transgenic plants; Environmental impact

\section{Introduction}

The interest in alternative strategies for controlling crop pests has been growing considerably in the world in response to the environmental and public health problems caused by the use of agrochemicals in crop protection. As a result of this raised public awareness, in Brazil, the public requests reliable information regarding the

\footnotetext{
${ }^{*}$ Corresponding author. Fax: +11-55-19-3867-8740/5531.

E-mail address: deise@cnpma.embrapa.br (D. Maria Fontana Capalbo).
}

commercial release, environmental consequences and food safety of genetically modified organisms (GMO). Consequently, the development of methods for assessing the effects of GMOs has increased in importance.

Public opinion is divided into those that favor the release of GMOs because they expect benefits from the possible reduction of food costs, increased yields and, hence, higher profits for the farmers, and those opposing the release of GMOs because of potential risks associated with these releases and the concern about the adequacy of the safety procedures for the environment and public health. Although the Brazilian government 
recognizes the difficulties involved, it promotes the research use, and labeling of transgenic products.

As indicated in the Cartagena Protocol on Biosafety of Living Modified Organisms (Biosafety Protocol) under the Convention on Biodiversity (CBD) and many other international forums, there is a clear need for comprehensive, transparent, scientific guidelines for meaningful pre-release testing and post-release monitoring of transgenic plants to ensure their environmental safety and sustainable use. The lack of such guidelines globally and the need for such guidelines in developing countries has been expressed repeatedly by both the private and public sector. Chapter 16 of Agenda 21 of the Cartagene Protocol on Biosafety of Living Modified Organisms recognizes that the maximum benefits of genetically modified crops can be achieved only if appropriate guidelines for their biosafety are in place and the relevant capacities to implement the guidelines are acquired.

\subsection{The GMO public perception in Brazil}

The same needs are recognized by the Brazilian Ministries of Agriculture, of Environment and of Science and Technology: to establish protocols dealing with economic aspects, public health, the conservation of biodiversity, scientific innovation, implicit technological dependencies and the vagaries of the import/export market.

In view of this, Embrapa, the Brazilian Agriculture Research Corporation, understood the importance of participating in a national and an international project to discuss and develop appropriate methods for the evaluation of potential environmental effects for the most relevant GMO products that Embrapa is developing.

Because of Embrapa's position, the need to strengthen risk assessments in Brazil, and the need to characterize and detect environmental impacts of Embrapa's GMOs that are presently under development, a group of Embrapa researchers accepted to participate in the international initiative called the "GMO Guidelines Project." It is an international initiative of public sector scientists organized within a global working group on 'Transgenic Organisms in Integrated Pest Management and Biological Control' under the umbrella of the International Organization of Biological Control (IOBC).

\subsection{The GMO guidelines project}

The GMO Guidelines Project (GGP) will develop scientific principles and detailed international scientific guidelines for biosafety testing of transgenic plants.

The key elements of this GGP are:

- An international initiative including expert scientists from leading research institutions from Europe, Australia, China, North America, East Africa, South America, and Southeast Asia.
- Coordination of the development and implementation of biosafety testing guidelines as a progressive, dynamic process, which will include scientific and technical capacity building and communication among scientists and between scientists and policy makers

- Swift, serial publication of sections of the guidelines as they are completed

- Rapid and timely revision of previously published sections should new scientific biosafety issues arise.

The GGP can be envisaged as a set of interlinked modules consisting of scientific questions related to risk assessment and corresponding scientific methodologies to answer those questions. The guidelines will have no regulatory legitimacy themselves, but regulatory authorities can choose to implement parts or all of the guidelines as they desire or need, with confidence in the scientific soundness behind the evaluations that follow the recommended methodologies. The guidelines will be designed for use on a case-by-case basis, as specified in the Biosafety Protocol and the European Union (EU) Directive on the release of GM plants. They will cover the environmental and agricultural impacts of GMOs, but they will not include the scientific expertise to evaluate human health impacts or ethical implications.

The GGP recognizes that a strong science base may be easier to transfer among countries than a regulatory system, consequently, the project focuses capacity building efforts on a few countries with reasonably developed scientific infrastructures. By strengthening the scientific capacities for risk assessment in these countries, it is expected that the necessary expertise can diffuse more readily to neighboring countries.

Project participants are either members of the core group or the project advisory board. The core group is divided into three regional groups and five scientific sections. The project is coordinated by the steering committee.

The core group is responsible for the development of the Guidelines that will be revised at regional workshops. The core group will be comprised of all IOBC Working Group members who express an interest and actively participate, and external resource people invited to complement group expertise.

The advisory board is invited to review periodically and provide advice on improving the products and process of the project. It is comprised of representatives from international and national organizations who have the scientific expertise and who can influence the international adoption of the guidelines. All members will be kept informed of the activities of the project via periodic communications and there will be one advisory board meeting. The scientific scope of the Guidelines has been divided into five scientific sections as follows:

\subsubsection{Needs analysis/good agricultural practice}

This section will provide a framework for evaluating the need for the transgenic plant in specific crop 
production contexts. This includes providing an approach to evaluating projected changes in crop production practices, such as tillage systems or insecticide use. It will incorporate a precautionary approach to the issues as specified by the Biosafety Protocol and EU legislation.

\subsubsection{Characterization of transgene construct and phe- notype in the plant}

This section will specify how a transgene should be described to enable evaluation of its stability and inheritance, and how the phenotypic effects of the transgene in the plant should be described; what, how, what plant parts, and when product concentrations should be measured in transgenic plants to facilitate assessment and management of environmental effects.

\subsubsection{Non-target effects}

This section will specify scientific procedures to determine the non-target species or function/process that should be tested (e.g., selection procedure) and scientific procedures for testing these species/functions/processes (e.g., testing procedure) for the following categories of organisms: natural enemies; pollinators; soil organisms; species of conservation concern; species of cultural significance; non-target pests; other non-target species.

Routes of exposure need to be identified. The organisms that are exposed need to be determined through suspected causal chains of impact. Based on this information, protocols, and methodologies for appropriate testing can then be developed.

\subsubsection{Pest-resistance management}

This section will specify procedures to determine the resistance risk of transgenic crops and feasible management responses needed to reduce this risk. It will also consider approaches for developing a practical monitoring and response system to detect resistance and to adapt management appropriately. While this is primarily addressing resistance development in insect pests, resistance development of weeds as a result of commercial planting of transgenic herbicide-tolerant crops will also be considered.

\subsubsection{Gene flow and its effects}

Gene flow is the route along which transgenes can spread genetically into populations of related species and geographically into other regions including protected areas of sensitive ecological value. Gene flow is considered a risk because of the great uncertainties associated with the possible consequences in the recipient ecosystems. Successful transgene flow will simultaneously affect both recipient plants and their associated organisms.

This section will determine protocols for establishing: (a) the likelihood of intra- and interspecific gene flow;

(b) the possibility of geographic and genetic spread of transgenes; (c) the potential ecological effects resulting from gene flow;

(d) the effectiveness of sterility mechanisms, their breakdown and management.

All public sector scientists are welcome to participate in the core group. Other interested people are invited to register on the mailing list of the project. An application form can be filled out on the project website http:// www.gmo-guidelines.info, be emailed, or printed and sent by air mail. One can also contact the project secretariat for application details (Evelyn Underwood $<$ underwood@geobot.umnw.ethz.ch>).

\subsection{Current situation of transgenic organisms in Brazil}

In Brazil more than 700 applications for field trials have been approved through 2001 . The vast majority of these field trials were carried out with corn ( $80 \%$ of these for demonstration purposes). Among the transgenic traits examined, the dominant ones were for resistance to herbicides followed by insect resistance (Bt-genes). The three main plant-trait combinations used in the field release trials were herbicide-resistant corn and soybean, and insect-resistant corn. Field trials also included more rare plant-trait combinations such as insect-resistant Btsoybean, herbicide-resistant Eucalyptus, insect-resistant Bt-sugarcane, herbicide-resistant rice, virus-resistant potato, and a number of insect-resistant corn cultivars whose traits were confidential.

The commercial production of Round-Up Ready soybeans (Monsanto) was initially approved in February 2002, by the Federal District Court. The approval, a six-hour oral presentation, was based on technical data presented to National Biosafety Technical Committee (CTNBio). It is important to mention that this matter has been under judicial discussion since 1998. After the initial approval in February 2002, the process continued, and no final decision has been made as of October 2002.

For more information about CTNBio (in Portuguese), visit http://www.ctnbio.gov.br/ctnbio/default.htm.

For more information on the project go to http:// www.gmo-guidelines.info.

\section{Acknowledgments}

The authors would like to acknowledge the Swiss Agency for Development and Cooperation that financed this project and all the members of the Global Working Group on Transgenic Organisms in Integrated Pest Management and Biological Control belonging to the International Organisation of Biological Control (IOBC), who supported and contributed to the project. The authors would also like to mention that the project receives no funding from the biotechnology industry. 